Felix Weil, Hektor Haarkötter:

\title{
Ethics for the Internet of Things
}

The Internet of Things (IoT) though being the latest major development in the digital sphere already has its own history. It was already in the early 1990s that Mark Weiser introduced the very idea of computing power embedded into entities in the physical world under his notion of Ubiquitous Computing. ${ }^{1}$ Later it was also discussed with slight modifications under the concepts of Pervasive Computing or Ambient Intelligence.

In 2007 IRIE has published an issue with the title: "Ethical Challenges of Ubiquitous Computing", edited by David Phillips and Klaus Wiegerling probing "the practices, ideologies, and power relations" of a "mesosphere saturated by information and communication technologies (ICT) ${ }^{\prime \prime}$. However, the very concept of an Internet of Things was originally proposed by Kevin Ashton in 1999 during a presentation at Procter \& Gamble in order to address the advent of the RFID technology making ordinary things seamlessly identifiable for remote computation. But it is only now after the huge enlargement of the IP address space by ip 6 and the further unabated rapid decline in costs of micro processing power that brings together the concepts of Ashton and Weiser to what we are willing to call now the very Internet of Things. This is fundamentally different to the idea associated with both approaches in particular but yet not less revolutionary. It is less the fact that computers today are embedded in more and more everyday items - from cars over mobile phones to TVs - that forms the IoT and thus the subject of this issue. And it is also not the remote computability of distinct physical (RFID tagged) items. The IoT is rather the being connected of embedded computing capability in finally everything that will form a more and more coherent digitally coined environment of our daily life.

In the course of the formation of the IoT our everyday world will thus become mantled and controlled by the capabilities of computers being embedded and connected to each other. our perceptions and actions, at alltime and everywhere, shall thus undergo some kind of ICT support. Everyday items will consequently not only be eyes and ears for computers but computers will also be connected to these everyday items resulting in hands of computers: billions of eyes, ears and hands to execute actions computers have decided upon which again are seen, heard and measured by other computers leading to further computed actions ... - thus, finally resulting in a Creative Circle (Varela) leading not to a new but separate (digital) sphere like the Internet of Websites (with a button to be switched of on every accessing device) but a complete new dimension of our existing physical world (that can't be switched of and thus be treated separately anymore as there is no distinct accessing device anymore): The very idea of an Internet of Things means an omnipresent ICT pervasion and accompaniment of our daily life, either as an active user, as a passive beneficiary, as a monitored and possibly even as a system guided being.

Basically, the IoT will consist of

- perception technology (sensors etc.) embedded in physical entities,

- networks for exchanging the data generated by these,

- computing power for interpreting this very very big data (in real time, as a service)

- and finally agents that react according to the computed results - the latter again embedded into everyday physical items being connected by the networks named above.

According to the research institution Gartner by 2020 more than 26 billion devices will be interconnected in this way - a multiple of human beings on the planet now and then. In a certain sense, our everyday world will be then made intelligent by the capabilities of computing power distributed and embedded into everyday objects and the connectivity of the net. 
There is no question that any technology that is going to so radically encroach on our daily life is in need of a robust ethical framework. Nevertheless, any ethical discussion of the Internet of Things rests inherently speculative because we are dealing with still emergent technology. We therefore have to take into account its full potential, irrespective of how far this potential can or will be realized in detail, and irrespective of the fields in which all-pervasive ICT accompaniment will find acceptance.

This brings into sharper focus two fundamental problems in theoretical ethics that have already attained a special position in applied media ethics in general and are now even more pressing in the IoT: On the one hand, the determination of reality which we should interpret for our moral decisions and which we should influence with our resulting acting. And on the other hand, the determination of the subject to which these actions should be attributed to and that should intervene with this reality. In the IoT we may say that on the one hand reality diminishes with respect to its (physical) confrontational character, and hence becomes more and more if not completely virtual or at least intrinsically virtually determined. The reality in the IoT won't be a sensational re-presentation of the physical world but more a virtual presentation involving and mantling it intrinsically physical and virtual at the same time to different degrees and extents in different situations which won't necessarily be transparent to the subjects involved.

And there comes into focus the subject that is perceived by intelligent systems always as a user stereotype, i.e. as a buying, sick or travelling subject etc. In a certain sense the subject in the IoT becomes weakened to the extent that some are willing to deny moral agency to the then computer enhanced/guided/discharged human beings and others are ready to ascribe moral agency not only to them alone but also to robots and computers interacting in the IoT; finally two ends of the same discussion. Yet, the status of the various agents in this virtual and interwoven reality is still to be clarified either regarding their moral accountability themselves (its degree like with adolescent human beings?) as well as its delegability (like from the product to its producer?).

Yet, looking deeper into the underlying developments it is not only the subject in certain situations but also its formation, the formation of its identity that is fundamentally affected by the IoT. This is because it has to above all manufacture its personality without the recognition and non-recognition of a present other genuine subject free of digital enhancement, and possibly without the development of those specific skills dependent on this confrontational experience of the 'naked' world and the 'naked' other. Our everyday respective abilities end up becoming substituted or at least adjusted by the intelligent systems underpinning it.

Thus, the experience of the world and the self will undergo a fundamental transformation in the IoT. At the possible end of this development it can happen what Ashton called the independence of the internet from any human intervention. The role models of active or passive participation in world affairs could then change dramatically, and the ethical dimensions of this transformation affect human actors as much as the "things". Most interestingly, the old English and German word "thing" etymologically meant a public assembly and therefore was a synonym of democracy and partizipation. The "Internet of Things" on the contrary will possibly become a notion of usurpation and the domination of computers over their former creators.

While this apocalyptic scenario portrayed in science fiction like 'Matrix' is to be considered as an extreme potential one thing remains for sure: things in cyber-physical systems - i.e. in the loT, i.e. in our future everyday world - won't remain the same as in pure physical reality today and thus we won't remain the same either as the relationship to the world is constitutive for the subject. What (sic!) things will be in the loT and how our selfunderstanding has to change accordingly is the very question at the bottom of any possible ethics for the loT.

\section{Questions to be asked; to be re-thought}

A possible and urgently necessary ethics for the phenomenon of an IoT is anchored in the field of information ethics, yet it radicalizes the fundamental issues in this area, insofar as the entire mesosphere appears as a sphere pervaded shaped and (in-)formed by virtual/computational facticities. Thus major issues of any ethics for the IoT are all yet addressed by infoethics but must be re-formulated and re-thought in the light of the above described radical developments: 


\section{Privacy in the IoT}

Of course, a major issue of the Internet of Things is privacy. As our everyday life will be invaded by sensors that are connected to computing power to process the 'Big Data' gathered the unprecedented possibilities to breach privacy are easily predictable. But again in the loT the quest for privacy is radicalized to the extent that the blurring of the contexts that define the realm of privacy and the public demands new fundamental concepts to define what these notions can really mean in the Internet of Things.

\section{Access to beneficial use of IoT and social justice}

Assuming that access to the Internet of Things is beneficiary for people and given its pervasiveness the potential making use of it may become a fundamental human right and constitutional for personal development. What do we have to do to avoid respective impairments and divides?

\section{Establishment of trust in the IoT}

The more our everyday life becomes dependent on the technologies deployed in the loT the more a framework is necessary to ethically establish trust in the loT. How can we and should we enable subjects to take informed decisions on attributing or depriving trust into the machinery.

\section{Status of agents and agency in the IoT}

In the case of the Internet of Things it is vital to clarify whether things that can act enabled by connected computing power are also actors from an ethical point of view. Can these things be attributed to some form of responsibility or accountability or only their originators? And how to regulate that?

\section{Answers given or outlined by the contributions of this issue}

The above named fundamental questions are explored by the contributions of this issue in different ways and dimensions:

- Ori Freiman asks if the concept of trust can provide a possible framework for constituting moral interaction in the IoT: Do we have to embed structures of trust into the things and their relationships as efficiently as we are embedding computing power into these? And how could this be achieved?

- Caroline Rizza and Laura Draetta are more sceptical regarding "technocratic approaches" to an ethics for the IoT. They argue, "that only human agency and user empowerment constitute a valid answer to the ethical, legal and social issues raised by IoT" and therefore demand a fundamental right to "silence the chips of IoT-things".

- Soenke Zehle is exploring a middle course by not opting for either silencing or unrestricted humming but proposing 'Depletion Design' as a fundamental design strategy to 'reclaim the ambient commons' in the IoT. "The idea of depletion design is ... to establish an experimental institutional object to facilitate and frame such ethico-aesthetic practice, an architecture for commoning that situates and affirms our ethical agency under the conditions of mediation."

- In their contribution Roba Abbas, Katina and M.G. Michael look more specific into the challenges Location-Based-Services are rising. It is a very comprehensive review of the respective techniques, their social application and the ethical challenges implicated. The authors finally propose a "socioethical conceptual framework" to address the fact that in the IoT "for the greater part, the human is removed from decision-making processes and is instead subject to a machine."

- Sandrina Dimitrijevic elaborates on the "Ethical Consequences of Bounded Rationality in the Internet of Things". She argues that any possible ethics for the IoT has to take into account that rationality in 
the IoT is by default bounded and we therefore cannot rely on informed consent alone as a last authority e.g. regarding privacy and giving away one's own data.

- Kashif Habib addresses the "Ethical Aspects of the Internet of Things in eHealth". While in his eyes "the healthcare system can get many benefits from the IoT such as patient monitoring with chronic disease, monitoring of elderly people ... [this] comfort may bring along some worries in the form of people's concerns such as right or wrong actions by things, unauthorised tracking, illegal monitoring, trust relationship, safety, and security." His paper presents the respective "ethical implications on people and society, and more specifically discusses the ethical issues that may arise due to distinguishing characteristics of the IoT."

- Bernhard Stengel presents "Ethical Thoughts Regarding Smart Homes". He also holds that smart technology may be more efficient than human beings in optimizing e.g. energy consumption but is also concerned about the underlying paternalism. What are the underlying norms for the optimization executed by these very efficient home automation systems?

- Burkhard Schafer sees "Data disposal as a challenge for waste management in the Internet of Things". The IoT will not only produce masses of e-waste we have to deal with in future but also dwaste: data stored on the billions of devices giving account of the everyday life of their users also and even more when disposed. Therefore, Schafer concludes: "Operators of large recycling schemes may find themselves inadvertently and unknowingly to be data controller for the purpose of Data Protection law, private resale of electronic devices can expose the prior owner to significant privacy risks." 